\title{
Development of Slow Control Package for the Electromagnetic Calorimeter Trigger System at the Belle II experiment
}

\author{
Cheolhun Kim ${ }^{\mathrm{a} 1}$, SungHyun Kim ${ }^{\mathrm{a} 2}$, InSoo Lee ${ }^{\mathrm{a} 3}$, HanEol Cho ${ }^{\mathrm{a} 4}$, YoungJun Kim ${ }^{\mathrm{b} 5}$, \\ JungKeun $\mathrm{Ahn}^{\mathrm{b} 6}$, Eunji Jang ${ }^{\mathrm{c}}$, SooKyoung Choi ${ }^{\mathrm{c} 8}$, Yuji Unno ${ }^{\mathrm{a} 9}$, ByungGu \\ Cheon ${ }^{\text {a10 }}$ \\ ${ }^{a}$ Dept. of Physics, Hanyang University, Seoul 04763, Republic of Korea \\ ${ }^{b}$ Dept. of Physics, Korea University, Seoul 02841, Republic of Korea \\ c Dept. of Physics, Gyeongsang National University, Jinju, 52828, Republic of Korea \\ E-mail: ${ }^{1}$ chkim@hep.hanyang.ac.kr, 2 sunghyun.kim@belle2.org, \\ ${ }^{3}$ islee@hep.hanyang.ac.kr, ${ }^{4}$ hecho@hep.hanyang.ac.kr, \\ ${ }^{5}$ rladudwns118@korea.ac.kr, 6 ahnjk@korea.ac.kr, \\ 7 jej744@gnu.ac.kr,8 schoi@gnu.ac.kr, \\ 9 yunno@post.kek.jp, 10 bgcheon@hanyang.ac.kr
}

In order to detect the new physics effect with heavy quark and lepton weak decays, the Belle II experiment held at the SuperKEKB in KEK(Japan) started Phase II, the second stage of the experiment, purpose of which are first beam collision test, sub-detector calbribration with beam and first physics data taking[1]. Belle II detector status have to be monitored for stable operation during trigger and DAQ operation upon beam collision. Slow control system, is one of main monitoring and controlling framworks of Belle II experiment, is based on NSM2(Network Shared Memory II) which is advanced version of NSM used at the Belle experiment. The technique let for all systems connected to Belle II server share data by memory sharing via network[2]. The ECL Trigger slow control system is the slow control system for Electomagnetic Calorimeter Trigger system. This system consists of 3 parts which are the user module, the configuration database and the archiver.

In this study, we report the development of slow control system for the Belle II electromagnetic calorimeter (ECL) trigger system during ongoing Phase II.

ICHEP 2018, International Conference of High Energy Physics

4-11 July 2018

Seoul, Korea

*Speaker

(C) Copyright owned by the author(s) under the terms of the Creative Commons 


\section{ECL Trigger Slow Control System}

The ECL trigger slow control system is for monitoring and controlling ECL trigger system which is one of indispensable sub-sytem of Belle II hardware during operation[3]. The system consists of the user module, the configuration database and the archiver parts.

\subsection{User module part}

The user module is built with Control System Studio(CSS) which is a GUI window design tool based on Eclipse. NSM2 variables and EPICS archived PVs can be shown on the module. It can also control the ECL trigger system through NSM2. Generating ready signal, reset experimental parameters of the system and initialization including rebooting command of the ECL trigger system are 3 main functions of this part. Other detailed variables and plots are also displayed.

\subsection{Configuration database part}

The configuration database is based on PostgreSQL. In the middle of Phase II, some of currently applied parameters on hardware such as firmware versions, FAM energy thresholds (576) and timing offsets (576) are stored on Belle II main database server, automatically. After firmware update, fitter-CC (303264) will be added. We also have to discuss which parameters should be stored more.

\subsection{Archiver part}

The archiver part is based on EPICS Archiver appliance and Apache Tomcat. NSM2 to EPICS conversion is well established. Some of ECLTRG outputs such as averaged hit-rate(4), FAM temperatures $(52 \times 2)$ and TMM temperatures $(7 \times 2)$ are archived on the Belle II main archiving server. Discussion is required to decide which data should be archived more. On the user module part, archived processed values(PVs) are displayed in real-time. Our archived PVs are also used for SuperKEKB machine tuning.

\section{Conclusion}

Infrastructures of slow control system which are the user module, database and the archiver are prepared. In the user module part, generating ready signal, reset and initialization command are included. Some of ECL trigger experimental paremters are stored on main the Belle II configuration database server, automatically. Several EPICS PVs are archived on the Belle II main archiving server. The PVs are not only for our system, but also for others such as SuperKEKB group. The ECL trigger slow control system should be updated for upcoming Phase III.

\section{References}

[1] T. Abe et al (Belle II Collaboration), Belle II Technical Design Rep. KEK Rep. 2010-1, Nov. 2010 [Online]. Available: http://arxiv.org/abs/1011.0352

[2] T. Konno et al., The Slow Control and Data Quality Monitoring System for the Belle II Experiment, IEEE Trans. Nucl. Sci., Vol. 62, No. 3, June 2015.

[3] S.H. Kim et al, "Status of the Electromagnetic Calorimeter Trigger system at the Belle II experiment”t, JINST 12 C09004, 2017 